\title{
Strong RFI Impact Mitigation In The Synthetic Aperture Interferometric Radiometer
}

\author{
Hyuk Park, Adriaon Camps, and Mercè Vall-llossera \\ CommSensLab \\ Universitat Politècnica de Catalunya and IEEC/CTE \\ Barcelona, Spain \\ park.hyuk@tsc.upc.edu, camps@tsc.upc.edu, \\ merce@tsc.upc.edu
}

\begin{abstract}
Radio Frequency Interference (RFI) is one of the problems causing the performance degradation in passive microwave radiometry. Especially, Synthetic Aperture Interferometric Radiometer (SAIR) is quite vulnerable to strong RFI. The Soil Moisture Ocean Salinity (SMOS) brightness temperature images show serious contamination by the RFI. The RFI affection in SAIR images should be mitigated or filtered out to retrieve the geophysical parameters. This work presents a method to RFI mitigation/filtering for SAIRs. Different from the existing method processing the brightness temperature image directly, RFI filtering of the subspace of covariance matrix is introduced, and the results are shown. The proposed method shows decent results for strong RFI with efficiency compared to the existing methods.
\end{abstract}

Keywords- Microwave radiometry; radio-frequency interference (RFI); RFI mitigation, soil moisture and ocean salinity (SMOS); synthetic aperture radiometry

\section{INTRODUCTION}

ESA Soil Moisture Ocean Salinity (SMOS) is the first and only mission that employed Synthetic Aperture Interferometric Radiometer (SAIR) payload. It was launched on 2 November 2009, and it is still providing the global maps of soil moisture and ocean salinity [1, 2]. In SMOS observation, Radio Frequency Interferences (RFI) are observed as decreasing the overall performance. RFI is still one of the barriers for synthetic aperture interferometric radiometer to overcome.

Thanks to the effort to report and turn off the RFI sources, many of them are removed. However, RFI sources are timevarying, and new RFI sources appear and disappear, which means they must be constantly monitored. Moreover, the RFI mitigation technique is required to improve the SAIR performance.

Synthetic Aperture Interferometric Radiometers (SAIR) such as SMOS have more serious contamination problem from RFI. Specifically, one strong RFI source easily contaminates a whole area of the brightness temperature $(\mathrm{Tb})$ snapshot. Therefore, a powerful mitigation method should be devised for SAIR, for current SMOS and future spaceborne SAIR mission.

\author{
Verónica González-Gambau \\ Institute of Marine Sciences, CSIC and the Barcelona \\ Expert Center \\ Barcelona, Spain \\ vgonzalez@icm.csic.es
}

Recently the sub-space method for SAIR imaging has been proposed, which deals RFI sources as strong signal measured from array system [3-5]. Based on the subspace of the covariance (visibility) matrix, the Direction-Of-Arrival of the RFI sources can be estimated in more accurately and precisely. The spatial filtering on the subspace is also applied for RFI filtering/mitigation of SAIR as well. The strong RFI sources have the large eigenvalues in the eigenvalue decomposition of the covariance matrix. Therefore, by filtering out (or suppressing) these large eigenvalues, the RFI mitigated covariance matrix is obtained, which finally generate the RFI mitigated brightness temperature.

In this work, the spatial filtering in covariance matrix used in the radio astronomy [6] is modified for the appropriate form to the Earth observation case like SMOS. Because of differences between two observation condition, the way in [6] cannot be directly used. The modified spatial filtering is tested by using several cases of SMOS RFI, e.g., single strong RFI, several RFI case, etc. Additionally, the mitigation of sun effect is also studied.

\section{MethODOLOGY}

From the viewpoint of array signal processing, the visibility samples of SAIR are equivalent to the elements of covariance matrix; cross-correlation between two elementary antenna outputs. Therefore, the covariance matrix of SAIR measurements can be constructed from visibility samples [5].

Once the covariance matrix $\mathbf{R}$ is constructed, it can be divided into the subspaces of the signal (RFI) and the noise (Tb) using the eigenvalue decomposition.

$$
\begin{gathered}
\mathbf{R}_{\boldsymbol{k}}=\left\langle\mathbf{y} \mathbf{y}^{\mathrm{H}}\right\rangle=\mathbf{A} \boldsymbol{\sigma}_{\mathbf{s}}^{2} \mathbf{A}^{\mathrm{H}}+\mathbf{a}_{\mathbf{k}} \sigma_{\mathrm{r}}^{2} \mathbf{a}_{\mathbf{k}}^{\mathrm{H}}+\boldsymbol{\sigma}_{\mathrm{n}}^{2}=\mathbf{R}_{\mathbf{s , n}}+\mathbf{R}_{\mathbf{R F I}} \\
=\left[\begin{array}{ll}
\mathbf{U}_{R F I} & \mathbf{U}_{s, n}
\end{array}\right]\left[\begin{array}{cc}
\boldsymbol{\Lambda}_{\boldsymbol{R F I}} & \mathbf{0} \\
\mathbf{0} & \boldsymbol{\Lambda}_{\boldsymbol{s}, \boldsymbol{n}}
\end{array}\right]\left[\begin{array}{ll}
\mathbf{U}_{R F I} & \mathbf{U}_{s, n}
\end{array}\right]^{H}
\end{gathered}
$$



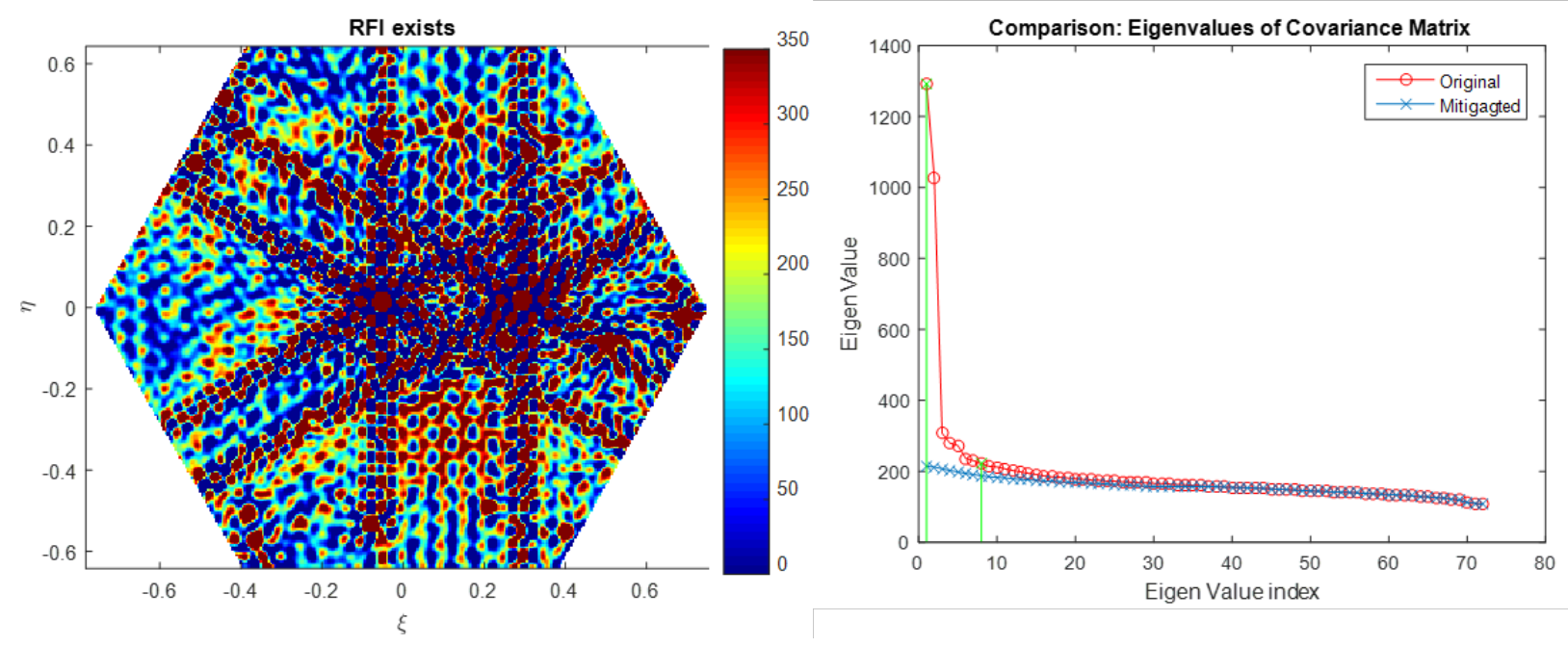

Fig. 1. RFI contaminated SMOS Tb snapshot (left) and corresponding eigenvalues of the covariance matrix (right).

where $\mathbf{A}$ is the steering matrix; $\boldsymbol{\sigma}_{\mathbf{s}}^{2}$ is the $\mathrm{Tb} ; \sigma_{\mathrm{r}}^{2}$ is the power of RFI source; and $\boldsymbol{\sigma}_{\mathrm{n}}^{2}$ is the noise power. The measured covariance $\mathbf{R}_{\mathbf{k}}$ can be divided into the RFI part $\mathbf{R}_{\mathbf{R F I}}$ and the Tb with noise part $\mathbf{R}_{\mathbf{s}, \mathbf{n}}$. By using the eigenvalue decomposition, the measured covariance matrix $\mathbf{R}_{\mathbf{k}}$ is divided into the RFI signal $\left(\mathbf{U}_{\mathrm{RFI}}\right)$ and Tb with noise $\left(\mathbf{U}_{\mathbf{s , n}}\right)$ subspaces; $\boldsymbol{\Lambda}_{\mathbf{R F I}}$ and $\boldsymbol{\Lambda}_{\mathbf{s , n} \mathbf{n}}$ are the diagonal matrices with eigenvalues of RFI signal subspace and Tb with noise subspace, respectively.

The number of RFI sources (diagonal dimension of $\boldsymbol{\Lambda}_{\mathrm{RFI}}$ ) is unknown and has to be estimated. For the SAIR RFI case, diagonal values of $\boldsymbol{\Lambda}_{\mathrm{RFI}}$ correspond to RFI sources powers are much larger than the diagonal values of $\boldsymbol{\Lambda}_{\mathbf{s}, \mathbf{n}}$. Using this property, the number of RFI source can be estimated.

An example RFI contaminated SMOS snapshot is shown in Fig. 1. As seen in the right column of Fig. 1, the eigenvalues of RFI power is much higher than the others (along with the red circles). In this example, the eigenvalues corresponding to the RFI source are considered up to the 8th one. The classification (or division) of eigenvalues between RFI sources and the other is explained in [4] in more detail.

In (1), RFI contributes via $\mathbf{U}_{\mathrm{RFI}}$ and $\boldsymbol{\Lambda}_{\mathrm{RFI}}$. The eigenvectors with high eigenvalue can be considered as an RFI components in the measured covariance matrix, as shown in Fig. 1. Then the RFI mitigation (or RFI filtering) can be approached by suppressing these high eigenvalues so as to become the comparable value to other eigenvalues $\boldsymbol{\Lambda}_{\boldsymbol{n}}$.

For the simple method, the RFI mitigated/filtered covariance matrix can be obtained by simple subtraction as

$$
\begin{aligned}
\mathbf{R}_{\mathbf{T b}} & =\mathbf{R}-\boldsymbol{\alpha} \mathbf{U}_{\mathrm{RFI}} \mathbf{U}_{\mathrm{RFI}}^{\mathrm{H}} \\
& =\left[\begin{array}{ll}
\mathbf{U}_{\mathrm{RFI}} & \mathbf{U}_{\mathrm{s}, \mathbf{n}}
\end{array}\right]\left[\begin{array}{cc}
\boldsymbol{\Lambda}_{\mathrm{RFI}}-\boldsymbol{\alpha} & \mathbf{0} \\
\mathbf{0} & \boldsymbol{\Lambda}_{\mathbf{s}, \mathbf{n}}
\end{array}\right]\left[\begin{array}{ll}
\mathbf{U}_{\mathrm{RFI}} & \mathbf{U}_{\mathrm{s}, \mathbf{n}}
\end{array}\right]^{\mathrm{H}}
\end{aligned}
$$

where $\boldsymbol{\alpha}$ is diagonal matrix suppress the high eigenvalues of RFI subspace. It compresses the diagonal values of matrix $\Lambda_{\mathrm{RFI}}-\boldsymbol{\alpha}$, making them in close to the diagonal values of $\boldsymbol{\Lambda}_{\mathbf{s}, \mathbf{n}}$.
In idea case, the diagonal elements of $\boldsymbol{\Lambda}_{\mathbf{s}, \mathbf{n}}$ are equal. However, it is not in the practical situation.

To find the suppressed term $\boldsymbol{\alpha}$, a simple algorithm is used in this study. In order to suppress the higher eigenvalues to be close to the values of others, those values are forced to be the average of other eigenvalues, i.e.

$$
\left(\boldsymbol{\Lambda}_{\mathrm{RFI}}-\boldsymbol{\alpha}\right) \approx \operatorname{mean}\left(\operatorname{diag}\left(\boldsymbol{\Lambda}_{\mathbf{s}, \mathbf{n}}\right)\right) .
$$

The RFI mitigated covariance matrix then has the much suppressed eigenvalues as shown in the second column of Fig. 1 . Along the blue crosses, the eigenvalues are mitigated and closed to their mean values.

Once the RFI mitigated covariance matrix $\mathbf{R}_{\mathbf{T b}}$ is obtained by compressing the eigenvalues of RFI subspace, the RFI mitigated brightness temperature image can be obtained using $\mathbf{R}_{\mathbf{T b}}$ instead of $\mathbf{R}_{\mathbf{k}}$ with the standard SMOS image reconstruction method.

\section{RESULTS}

Fig. 3 shows two examples of RFI mitigation results. The actual measured SMOS Tb images in the middle are seriously contaminated by the RFI. Specifically, one or two very strong RFI sources are contaminating the whole image with their long tails and many dimples. Those strong sources have even around $\sim 1000 \mathrm{~K}$. Therefore, whole regions of these snapshots cannot be used for $\mathrm{Tb}$ estimation.

These strong RFI sources are well revealed as eigenvalues of covariance matrix as shown in the first column in Fig. 3. The higher eigenvalues in original (red circles) are suppressed in the mitigated ones (blue crosses). 

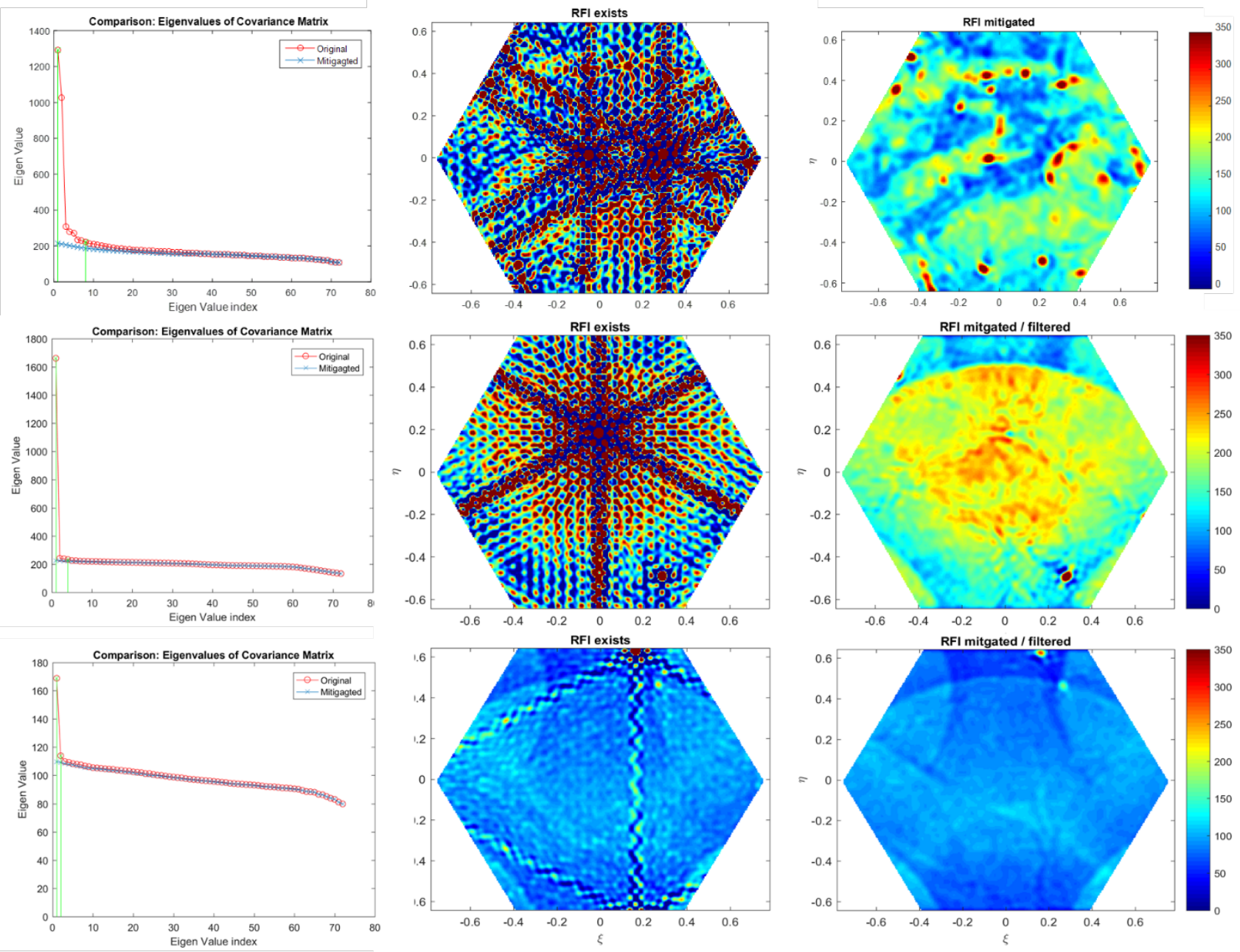

Fig. 2 Results of SMOS RFI mitigation: eigenvalue pots of covariance matrices of the original and mitigated ones (left column), RFI contaminated brightness temperature images (middle column), RFI mitigated TB images (right column). The first row shows the case where RFI sources are located on coastline, the second row for the case of in the land, and the third rows for the sun effect over the ocean.

The images on the right are RFI mitigated by using the proposed eigenvalue suppression. Although hot spots still exist, the RFI effects are significantly removed, i.e. there are no tails around strong RFI sources. Also, the sidelobe effects are removed.

The first row in Fig. 2 is the cases of strong RFI sources located coastline. There are two strong RFI sources contaminating the whole area, which prevent to recognize the land and sea. After the mitigation, it shows the coastal line between the land and sea. The second row in Fig. 2 is the case of strong RFI source located in the land. It also shows the much RFI mitigated results.

The third row if Fig. 2 shows the sun effect over the ocean. The sun is seen in uppermost part in Fig. 2 (third row, second column). Although the eigenvalue is not very high (165 in Fig. 2 , eigenvalue plot), it contaminates $\mathrm{Tb}$ image over the ocean which is relatively low $\mathrm{Tb}(\sim 120 \mathrm{~K})$. The tails and sidelobes are severe in the Tb snapshot. After the mitigation, these effects are much reduced. Therefore, the method can be used not only the RFI source, but also any strong signal source such as sun effect.

\section{DISCUSSIONS}

For the simple subtraction method used here, it can be interpreted as the projection filtering. The subtraction in (2) can be considered as

$$
\begin{aligned}
\mathbf{R}_{\mathrm{Tb}} & =\mathbf{R}_{\mathbf{k}}-\boldsymbol{\alpha} \mathbf{U}_{\mathrm{RFI}} \mathbf{U}_{\mathrm{RFI}}^{\mathrm{H}} \\
& =\left(\mathrm{I}-\boldsymbol{\alpha}^{\prime} \mathbf{U}_{\mathrm{RFI}} \mathbf{U}_{\mathrm{RFI}}^{\mathrm{H}}\right) \mathbf{R}_{\mathbf{k}}\left(\mathbf{I}-\boldsymbol{\alpha}^{\prime} \mathbf{U}_{\mathrm{RFI}} \mathbf{U}_{\mathrm{RFI}}^{\mathrm{H}}\right) \\
& =\mathbf{P} \mathbf{R}_{\mathbf{k}} \mathbf{P} \approx \mathbf{P} \mathbf{R}_{\mathbf{s}, \mathbf{n}} \mathbf{P}
\end{aligned}
$$

where $\mathbf{P}$ is the projection matrix for filtering the strong signal. In this case, the subtraction can be represented by a two-sided linear operation, and the Tb covariance matrix $\mathbf{R}_{\mathbf{s , n}}$ is altered. Therefore, the recovering process is required to obtain it, using the property of Kronecker product as [6]:

$$
\begin{aligned}
\widehat{\mathbf{R}}_{\mathbf{s}, \mathbf{n}} & \equiv \operatorname{unvec}\left(\mathbf{C}^{-\mathbf{1}} \operatorname{vec}\left(\mathbf{R}_{\mathbf{k}}\right)\right) \\
\mathbf{C} & =\left(\mathbf{P}_{\mathbf{k}}^{\mathbf{T}} \otimes \mathbf{P}_{\mathbf{k}}\right)
\end{aligned}
$$


where $\otimes$ denotes the Kronecker product. Therefore, the RFI mitigated covariance matrix $\widehat{\mathbf{R}}_{\mathbf{s}, \mathbf{n}}$ is obtained, and the RFI mitigated $\mathrm{Tb}$ image is reconstructed using it.

However, it is difficult to directly employ the method in (4) and (5) for satellite earth observation. The problem is the invertibility of $\mathbf{C}$ matrix in (5). The $\mathbf{C}$ matrix is invertible in conditions of varying the RFI source condition (directions) in many snapshots. The satellite SAIR like SMOS is not exactly the same conditions, and therefore a special adaptation/ modification is required, which will be further studied.

\section{CONCLUSIONS}

In order to improve the performance of the microwave radiometry, RFI problems should be properly managed. Not only source detection and geolocation, the RFI mitigation methods have to be developed to effectively cope with temporally and spatially varying characteristics of RFI sources. This work presents a SAIR RFI mitigation based on the subspace of covariance matrix. The eigenvalue suppression method is proposed with some example results. The result shows the Tb images in which strong RFI sources are much suppressed. This method is efficient compared with iteration method using Tb image.

\section{ACKNOWLEDGMENT}

This work was supported by the Spanish Ministry of Economy and Competitiveness and FEDER EU under the project "AGORA: Tecnicas Avanzadas en Teledeteccion Aplicada Usando Senales GNSS y Otras Senales de Oportunidad” (MINECO /FEDER) ESP2015-70014-C2- 1-R, PROMISES: Productos y servicios innovadores con sensors de microondas, SMOS y Sentinels para tierra ESP2015-67549-C31-R, ESP2015-67549-C3-2-R; and financed by the Agencia Estatal de Investigación, Spain. Unidad de Excelencia Maria de Maeztu MDM-2016-0600.

\section{REFERENCES}

[1] J. Font, A. Camps, A. Borges, M. Martin-Neira, J. Boutin, N. Reul, Y. H. Kerr, A. Hahne, and S. Mecklenburg, "SMOS: The Challenging Sea Surface Salinity Measurement From Space,” Proc. IEEE, vol. 98, no. 5, pp. 649-665, 2010.

[2] SMOS-BEC, CP34-BEC, SMOS-BEC data distribution and visualization services. [Online]. Available: http://cp34-bec.cmima.csic.es

[3] H. Park, V. Gonzalez-Gambau, and A. Camps, "High Angular Resolution RFI Localization in Synthetic Aperture Interferometric Radiometers Using Direction-of-Arrival Estimation,” IEEE Geosci. Remote Sens. Lett., vol. 12, no. 1, pp. 102-106, 2015.

[4] H. Park, V. Gonzalez-Gambau, A. Camps, and M. Vall-llossera, "Improved MUSIC-Based SMOS RFI Source Detection and Geolocation Algorithm,” IEEE Trans. Geosci. Remote Sens., pp. 1-12, 2015.

[5] H. Park, V. Gonzalez-Gambau, A. Camps, and M. Vall-llossera, "Feasibility of RFI Mitigation in Synthetic Aperture Radiometry Based On Subspace Spatial Filtering,” IGARSS 2017, 2017, Jul, Fortworth. USA.

[6] A.-J. Boonstra, A.-J. van der Veen, and J. Raza, "Spatial filtering of continuous interference in radio astronomy," in IEEE International Conference on Acoustics Speech and Signal Processing, 2002, p. III2933-III-2936. 